\title{
ROUND HANDLES AND HOMOTOPY OF NONSINGULAR VECTOR FIELDS
}

\author{
BY DANIEL ASIMOV
}

Communicated by S. S. Chern, October 24, 1974

Introduction. We consider nonsingular vector fields on compact connected $C^{\infty}$ manifolds. The question is: What orbit structures occur in which homotopy classes of nonsingular vector fields? We show that in dimensions 4 and greater, a nonsingular Morse-Smale (NMS) vector field occurs in each homotopy class. Under the additional assumption that the first Betti number of the manifold is nonzero, we show that a nonsingular volume-preserving (NVP) vector field occurs in each homotopy class. These results are based on the round handle decomposition theorem, interesting in its own right as a structure theorem for manifolds whose Euler characteristic is 0 .

Let $M$ be a compact manifold whose boundary has been partitioned into two unions of components: $\partial M=\partial_{-} M \cup \partial_{+} M, \partial_{-} M \cap \partial_{+} M=\varnothing$. Then the following are equivalent:

1. $\chi\left(\partial_{-} M\right)=\chi(M)$.

2. $\chi\left(\partial_{+} M\right)=\chi(M)$.

3. There exists a nonsingular vector field on $M$ pointing inward on $\partial_{-} M$ and outward on $\partial_{+} M$.

Definition. The pair $\left(M, \partial_{-} M\right)$ will be called a flow manifold if 1,2 and 3 above are true. This does not exclude the possibility, of course, that $\partial_{-} M, \partial_{+} M$, or $\partial M$ may be empty.

Definition. A nonsingular Morse-Smale (NMS) vector field $V$ on the flow manifold $\left(M, \partial_{-} M\right)$ is one which satisfies (a), (b) and (c) below:

(a) $V$ has nonwandering set equal to a finite number of closed orbits, each having a hyperbolic Poincaré map.

(b) The stable manifold (inset) of one closed orbit is transversal to the unstable manifold (outset) of any other closed orbit.

(c) $V$ points inward on $\partial_{-} M$ and outward on $\partial_{+} M$.

Definitions. A round handle of index $k$ (and dimension $n$ ) is a copy

AMS (MOS) subject classifications (1970). Primary 57D25. 
of $R_{k}=S^{1} \times D^{k} \times D^{n-k-1}$. The attaching region $\partial_{-} R_{k}$ of $R_{k}$ is the submanifold $S^{1} \times S^{k-1} \times D^{n-k-1}$ of $\partial R_{k}$, where $S^{k-1}=\partial D^{k}$.

THEOREM 1 (ROUND HANDLE DECOMPOSITION THEOREM). Let $\left(M^{n}, \partial_{-} M\right)$ be a flow manifold with $n \geqslant 4$. Then M admits a decomposition of the form

$$
M=\left(\partial_{-} M \times I\right)+R_{0}^{1}+\cdots+R_{0}^{\alpha} 0+R_{1}^{1}+\cdots+R_{n-1}^{\alpha}{ }_{n-1} .
$$

This means that each $R_{k}^{i}$ is attached via a diffeomorphism of $\partial_{-} R_{k}^{i}$ to the boundary of the previous stuff, but never to $\partial_{-} M \times\{0\}$. If we further assume that $\partial_{-} M \neq \varnothing$ and $\partial_{+} M \neq \varnothing$, we may write

$$
M=\left(\partial_{-} M \times I\right)+R_{1}^{1}+\cdots+R_{n-2}^{\beta}{ }_{n-2},
$$

i.e. there exists a round handle decomposition avoiding round handles of extreme indices.

CoROllary 1. Let $\left(M^{n}, \partial_{-} M\right)$ be a flow manifold with $n \geqslant 4$. Then $\left(M, \partial_{-} M\right)$ admits an NMS vector field. If we further assume that $\partial_{-} M \neq \varnothing$ and $\partial_{+} M \neq \varnothing$, then $\left(M, \partial_{-} M\right)$ possesses an NMS vector field with no source or sink closed orbits.

THEOREM 2. Let $V$ be a nonsingular vector field on the flow manifold $\left(M^{n}, \partial_{-} M\right)$ pointing inward on $\partial_{-} M$ and outward on $\partial_{+} M$. Assume $M$ is orientable and $n \geqslant 4$. Then $V$ is homotopic rel $\partial M$ (through nonsingular vector fields) to an NMS vector field. If further $\partial_{-} M \neq \varnothing$ and $\partial_{+} M \neq \varnothing$ then $V$ is homotopic rel $\partial M$ to an NMS vector field with no sources or sinks.

THEOREM 3. Let $M^{n}$ be compact, connected, and orientable with $\chi(M)$ $=0, n \geqslant 4$, and $H^{1}(M) \neq 0$. Then any nonsingular vector field $V$ on $M$ is homotopic to a field $V_{1}$ which preserves some smooth nonzero volume form $\omega$ on $M$ (which may be preassigned).

Proof (SKetch). Using $H^{1}(M) \neq 0$ we first find a compact, connected, oriented submanifold $i$ : $N^{n-1} \subseteq M$ with $i_{*}[N] \neq 0$ in $H_{n-1}(M)$. By surgering $N$ we may find a homologous submanifold $N^{\prime}$ and a vector field $V^{\prime}$ homotopic to $V$ such that $V^{\prime}$ is nowhere tangent to $N^{\prime}$. Then cutting $M$ open along $N^{\prime}$ gives us a flow manifold $\widetilde{M}$, and $V^{\prime}$ becomes a vector field $\widetilde{V}$ on $\widetilde{M}$. By Theorem 2, $\widetilde{V}$ is homotopic rel $\partial \widetilde{M}$ to an NMS vector field $\widetilde{V}_{1}$ on $\widetilde{M}$ having no sources or sinks. This last property permits finding (one round handle at a time) a smooth nonzero volume $\widetilde{\omega}$ on $\widetilde{M}$ preserved by $\widetilde{V}_{1}$. By carefully regluing 
$\widetilde{M}$ to obtain $M$ once again, $\widetilde{V}_{1}$ becomes a smooth nonsingular vector field $V_{1}$ (which is not NMS), and $\widetilde{\omega}$ becomes a smooth nonzero volume $\omega$ on $M$ preserved by $V_{1}$. The form $\omega$ may as well have been preassigned, since Moser [4] shows any two volume forms are equivalent up to constant multiple under a diffeomorphism of $M$ isotopic to the identity.

Remark. Theorem 3 fails on the 2-torus $T^{2}$.

\section{REFERENCES}

1. D. Asimov, Flow manifolds, Thesis, University of California, Berkeley, Calif., 1972.

2. - Homotopy of nonsingular vector fields (preprint).

3. M. A. Kervaire, Le théorème de Barden-Mazur-Stallings, Lecture Notes in Math., vol. 48, Springer-Verlag, Berlin and New York, 1967, pp. 83-95.

4. J. Moser, On the volume elements on a manifold, Trans. Amer. Math. Soc. 120 (1965), 286-294. MR 32 \#409.

5. B. L. Reinhart, Cobordism and the Euler number, Topology 2(1963), 173-177. MR 27 \#2990.

6. S. Smale, Morse inequalities for a dynamical system, Bull. Amer. Math. Soc. 66 (1960), 43-49. MR 22 \#8519.

7. - Differentiable dynamical systems, Bull. Amer. Math. Soc.. 73 (1967), 747-817. MR 37 \#3598; erratum; 39, 1593.

8. - Stability and isotopy in discrete dynamical systems, Dynamical systems (edited by Peixoto), Academic Press, New York, 1973, pp. 527-530.

9. M. Shub, Stability and genericity for diffeomorphisms, dynamical systems, Academic Press, New York, 1973, pp. 493-514.

10. - Morse-Smale diffeomorphisms are unipotent on homology, dynamical systems, Academic Press, New York, 1973, pp. 489-491.

SCHOOL OF MATHEMATICS, UNIVERSITY OF MINNESOTA, MINNEAPOLIS, MINNESOTA 55455 